\title{
Lessons from arrhythmogenic right ventricular cardiomyopathy research
}

\author{
Matthew Taylor, MD, PhD
}

$\mathrm{H}$ uman Mendelian disease researchers must frequently balance research priorities and the need to validate research data with the pressures to apply their new knowledge quickly to the clinical arena to satisfy clinicians and affected patients and families. Striking the correct balance between clinical and research priorities, in the spirit of Goldlilocks' pursuit to get things "just right," is not always easy and often represents a moving target for researchers and clinicians. The continuing story unfolding for arrhythmogenic right ventricular cardiomyopathy (ARVC) provides one of the best illustrations of how perceived clinical needs have continually motivated and at times pressured the pace of research agendas.

Somewhat remarkably, the ARVC phenotype captured the attention of genetic researchers relatively recently, even though the Pope's physician Giovanni Maria Lancisi described the condition in a multigenerational family in 1736. ARVC is common for a Mendelian disease (1 in 1000 to 1 in 5000 individuals affected); however, comprehensive descriptions of the phenotype were largely not offered until the early 1980s. Like other cardiomyopathies, ARVC is a primary disease of the myocardial tissue that shows a dominant pattern of inheritance. Pathologically, progressive replacement of normal myocardium with fibro-fatty tissue leads to arrhythmias, heart failure, and sudden cardiac death. The disease presents in young adults who often have an unremarkable personal medical history. It may be more frequent in high-performance athletes suggesting that some element of exercise physiology may be important in bringing out the phenotype. The presentation of intermittent palpitations in otherwise healthy, vigorous, young persons can be diagnostically challenging for clinicians who will often have a low a priori suspicion for a lethal cardiac condition in a young population.

Diagnostic criteria have been developed that include abnormalities in structure and function of the right ventricle (assessed by echocardiography), conduction and arrhythmias (electrocardiography and continuous monitoring), tissue histology, and family history. ${ }^{1}$ The diagnosis in an index patient can be difficult because each diagnostic modality suffers from limited sensitivity; even repeated cardiac biopsies can accidentally "miss" the somewhat patchy right ventricular involvement, especially in early stage disease. Because cardiac biopsies do include a procedural risk, they are not always done early in the workup of an index patient. An analysis of the family history may reveal individuals with unexplained sudden death in whom autopsies were not performed or where the cardiac autopsy

From the Department of Medicine, Division of Cardiology, Adult Medical Genetics Program, University of Colorado Denver, Aurora, Colorado.

Matthew Taylor, MD, PhD, 12700 East 19th Avenue, Mailstop F442, Room 8022, Aurora, CO 80045. E-mail: matthew.taylor@ucdenver.edu.

Disclosure: The author declares no conflict of interest.

Submitted for publication October 23, 2009

Accepted for publication October 26, 2009.

DOI: 10.1097/GIM.0b013e3181c7ef3b analysis was restricted more toward coronary anatomy analysis than a careful assessment of right ventricular problems. How to classify "suspicious" findings in the family history that do not meet strict criteria require clinical judgment. One careful analysis of at-risk relatives led to the suggestion that diagnostic criteria for patients who were not probands could include more relaxed criteria to increase sensitivity to account for these diagnostic challenges and to reduce the likelihood of a diagnostic false negative in a family that has already declared itself to have ARVC. ${ }^{2}$ Thus, the landscape for ARVC researchers was a challenging one from the outset. Faced with a disease that is genetic, lethal, difficult to diagnose, and equally difficult to exclude, it was critical to identify ARVC genes and provide risk assessment to families. Beyond the genetic counseling needs, ARVC differed from many other Mendelian diseases because interventions and therapies, including prophylactic defibrillator implantation, were possible even before the genetic basis was completely understood. The lethality of the phenotype, the inadequacy of available clinical testing, and the potential for interventional therapy conspired to make separation between provocative research questions and clinically urgent needs difficult at best.

It is on this background that the article by Drs. Hodgkinson and Dicks ${ }^{3}$ provides important context and insight into how the interplay of ARVC research and clinical agendas unfolded in the Newfoundland and Labrador. An ARVC founder mutation was suspected in this population and this hypothesis was validated when a causative mutation in TMEM43 was detected in multiple ARVC families, paving the way for early and presymptomatic diagnosis. Parallel research by their group further delineated the phenotype as being frequently lethal, more severe in males, and included heart failure in older-surviving adults. Even before the localization of the exact mutation, they demonstrated improved survival with defibrillator therapy in individuals with the pathogenic ARVC haplotype. A consequence of this important work was that clinical decisions and management approaches were being applied simultaneously with steady advances on the research front. Not surprisingly, problems and conflicts arose with ethical considerations, confidentiality protocols, and the adjudication of research data that was then applied toward clinical goals. Although institutional review boards often try to define boundaries between research and clinical efforts, this article eloquently illustrates the fluid and unpredictable nature of this interface.

Of the six lessons expounded in the article, two are worth reviewing here. The first, Lesson 2, is that diseases like ARVC require a close working relationship between clinical and research genetics. Barriers to sharing research data including "clinical tests" obtained for research such as echocardiograms and electrocardiograms were present and led to disastrous consequences in some cases. Inconsistency on this point likely exists between different research institutions where clinical test data obtained for research purposes are deposited in the medical records of some but not others. The authors pointed out that some encountered barriers resulted from unwillingness of USbased researchers to share some of these data due to real or 
perceived conflicts with releasing data with the "research" label attached. The discovery of a disease-associated ARVC haplotype by the research group further complicated matters because it carried a high sensitivity for detecting clinically at-risk individuals but was offered only through a research laboratory. This likely reversed the tables for the group, putting the US collaborators in a difficult position because non-Clinical Laboratory Improvement Amendments-approved tests cannot be used for clinical care in the United States, even when they ironically represent the best available test.

The second lesson that institutional review boards and others should take note of is Lesson 4 arguing that circumstances exist where patients should be considered both as research subjects and clinical patients. One of the seemingly contradictory aspects of institutional review boards is a charge to disclose the planned and anticipated outcomes of clinical research projects even though the purpose of many research projects is the discovery of new knowledge and paradigms that are poorly predicted before initiation of research. Although the Newfoundland and Labrador ARVC project was organized initially around the question of ARVC etiology, it rapidly evolved a number of clinical foci that blurred the distinction between research and clinical subjects. In one instance, a young man whose malignant ARVC haplotype was known to investigators refused to hear his "research" results; he subsequently died. Review board policies are not solely to blame here, as a long history of nondirectiveness in genetics can also leave a genetics researcher uncertain on how far to venture into the area of clinical management and when to assume a directive role in providing clinical advice and recommendations. The lessons in this article are not restricted to ARVC and have relevance to other genetic conditions where clinical questions and needs are closely related to ongoing research. As understanding of disease pathogenesis moves forward, presumably the opportunity to apply sensible treatments based on some level of research data will become more commonplace and the lessons presented in this work more generalizable.

\section{REFERENCES}

1. McKenna WG, Thiene G, Nava A, Fontaliran C, Blomstrom-Lundqvist G. Diagnosis of arrhythmogenic right ventricular dysplasia/cardiomyopathy. $\mathrm{Br}$ Heart J 1994;71:215-218.

2. Hamid MS, Norman M, Quraishi A, et al. Prospective evaluation of relatives for familial arrhythmogenic right ventricular cardiomyopathy/dysplasia reveals a need to broaden diagnostic criteria. J Am Coll Cardiol 2002;40:1445-1450.

3. Hodgkinson K, Dicks E, Connors D, et al. Translation of research discoveries to clinical care in arrhythmogenic right ventricular cardiomyopathy in Newfoundland and Labrador: Lessons for health policy in genetic disease. Genet Med 2009;11:860-866. 